\title{
An optical tweezer-based study of antimicrobial activity of silver nanoparticles
}

\author{
YOGESHA, SARBARI BHATTACHARYA, M K RABINAL ${ }^{\dagger}$ and SHARATH ANANTHAMURTHY* \\ Department of Physics, Bangalore University, Bangalore 560 056, India \\ ${ }^{\dagger}$ Department of Physics, Karnatak University, Dharwad 580 003, India
}

MS received 19 February 2012; revised 29 March 2012

\begin{abstract}
Understanding and characterizing microbial activity reduction in the presence of antimicrobial agents can help in the design and manufacture of antimicrobial drugs. We demonstrate the use of an optical tweezer setup in recording the changes in bacterial activity with time, induced by the presence of foreign bodies in a bacterial suspension. This is achieved by monitoring the fluctuations of an optically trapped polystyrene bead immersed in it. Examining the changes in the fluctuation pattern of the bead with time provides an accurate characterization of the reduction in the microbial activity. Here, we report on the effect of addition of silver nanoparticles on bacterial cultures of Pseudomonas aeroginosa, Escherichia coli and Bacillus subtilis. We observe a decrease in the bacterial activity with time for the investigated bacterial samples. This method in our opinion, enables one to track changes in bacterial activity levels as a function of time of contact with the antibacterial agent with greater efficacy than traditional cell counting methods.
\end{abstract}

Keywords. Antimicrobial activity; optical tweezer; bacterial suspensions; silver nanoparticles.

\section{Introduction}

The toxicity of silver ions and silver containing compounds on microbes is well known. Nanoparticles of silver are expected to exhibit enhanced antimicrobial properties when compared to the bulk metal due to a much larger surface area to volume ratio. Various studies suggest that heavy metals like $\mathrm{Ag}$ react with proteins by combining the $-\mathrm{SH}$ groups of enzymes, resulting in their inactivation thus leading to bacterial growth inhibition (Lehninger and Nelson 1993). Feng et al (2000) studied the antibacterial action of silver ions on E. coli and S. aereus. Their study showed that the effect of silver ions was to transform the free state of DNA molecules to a condensed form causing them to lose their replication abilities. Their study also suggests that the interaction of $\mathrm{Ag}$ ions with thiol groups in proteins renders inactivation of bacteria (Feng et al 2000).

Bactericidal efficiency of antibacterial agents is generally gauged through disk diffusion tests, minimum inhibitory concentration (MIC) and minimum bactericidal concentration (MBC) measurements (Ruparelia et al 2008). Estimation of these effects can also be carried out by optical density measurements with UV-visible spectrometers or measurement of colony formation units using colony counters (Raffi et al 2008). In this work, we demonstrate the use of an optical tweezer in monitoring the effect of antibacterial action of silver nanoparticles in suspensions of different strains. Through characterizing their activity and monitoring the same in time,

\footnotetext{
*Author for correspondence (asharath@gmail.com)
}

we are able to provide a real time assessment of effect of the antimicrobial agent as the measurements are made continuously on the same bacterial bath. This is in contrast to cell counting methods that require time consuming repeated measurements on fresh and (desirably) identical samples.

\section{Experimental}

\subsection{Growth of bacterial strains}

We chose Pseudomonas aeroginosa, Escherichia coli and Bacillus subtilis for our studies. Pseudomonas aeroginosa and Escherichia coli are gram-negative opportunistic human pathogens while Bacillus subtilis is a gram positive bacterium. Under a microscope they all appeared rod-like in shape.

Overnight cultures were diluted in sterilized nutrient broth, which was a mixture of $3.0 \mathrm{~g}$ beef extract, $5.0 \mathrm{~g}$ peptone, $5.0 \mathrm{~g} \mathrm{NaCl}$ and 1 litre distilled water. The cultures in the nutrient broth were then grown at $37^{\circ} \mathrm{C}$ until cell counts reached a stationary phase. The silver nanoparticles were then added to the cultures and mixed uniformly by agitating for a few minutes. About $1-2 \mu \mathrm{l}$ of $3 \mu \mathrm{m}$ diameter sterile polystyrene beads were then added to the cultures under investigation. The measurements were carried out immediately after this and then again after certain desired time intervals. During the measurements, care was taken to maintain a constant distance of $30 \mu \mathrm{m}$ between the trapped bead and cover slip to avoid surface effects. 


\subsection{Silver nanoparticle synthesis}

Silver nanoparticles capped with starch molecules were prepared by wet chemical route. Details of synthesis and characterization of the silver nanoparticles will appear elsewhere (Rabinal et al 2012). In brief, stock solution of $0.5 \mathrm{~g}$ starch was prepared in $100 \mathrm{ml}$ of distilled water. $1 \mathrm{M}$ of $\mathrm{AgNO}_{3}$ was added to $5 \mathrm{ml}$ of this stock solution and then ultrasonicated for more than $15 \mathrm{~min}$. The prepared solution was then exposed to short wavelength UV radiation $(\lambda=253 \mathrm{~nm})$ for $20 \mathrm{~min}$ in a home-made setup, resulting in a colour change from clear to brown indicating formation of silver nanoparticles. We estimated the average nanoparticle size by surface plasmon resonance spectra measured by a visible-NIR CCD spectrometer (Alvarez et al 1997). The particle size was found to be $5 \mathrm{~nm}$ (figure 1).

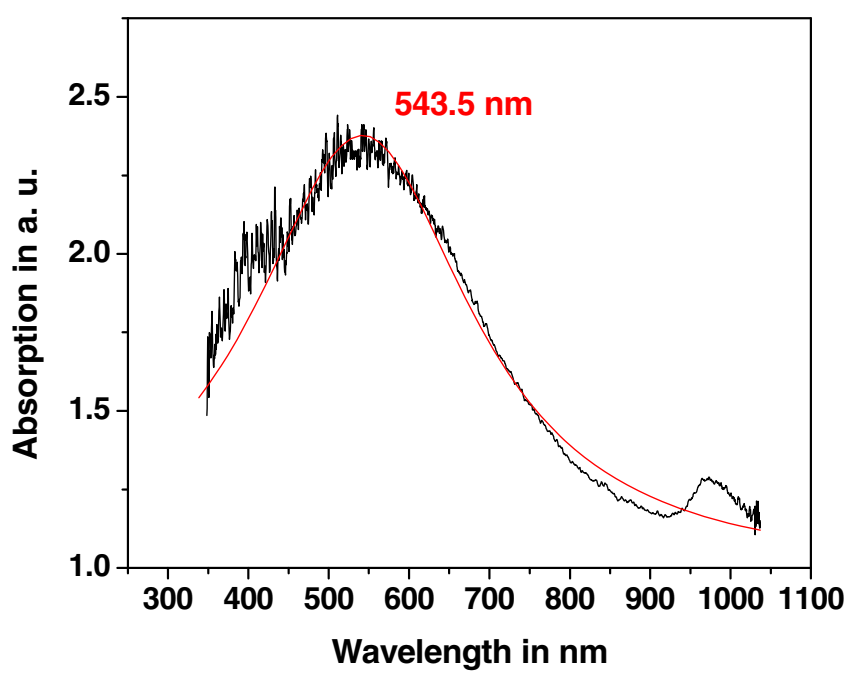

Figure 1. Absorption spectrum measured for silver nanoparticles solution. Solid line is a fit to a Lorentzian.

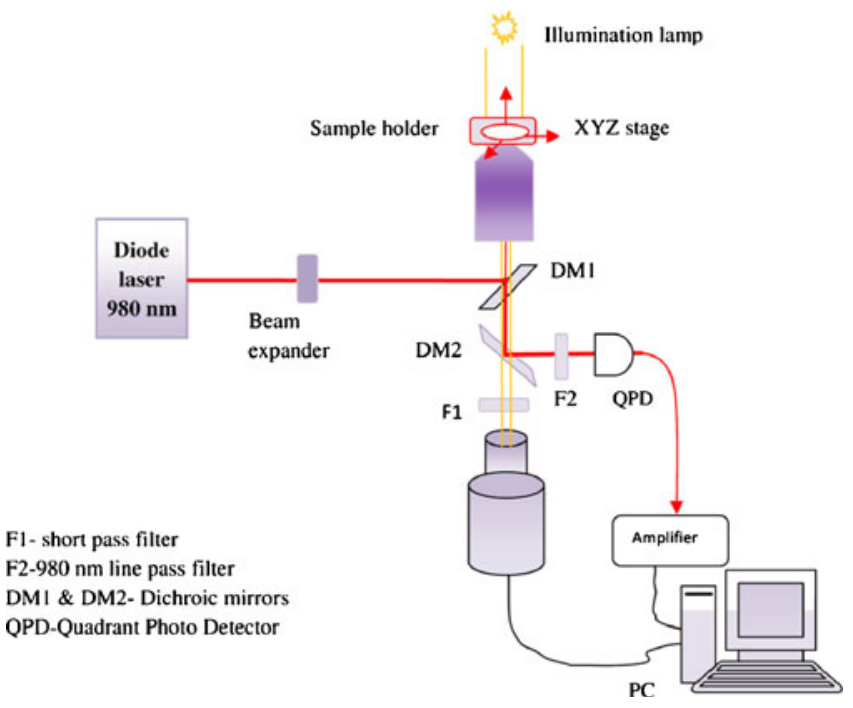

Figure 2. Optical tweezer-A schematic view.

\section{Optical tweezers}

The optical tweezer setup used by us has been described elsewhere (figure 2) (Yogesha et al 2011). Briefly, it consists of a trapping diode laser $(980 \mathrm{~nm})$ guided into an oil immersion objective (1.4 NA, EA100 $\times$ ) on a custom made inverted microscope. The instrument is also equipped with a PZT stage and a quadrant photo detector (QPD) for laser beam detection, which in turn measures the position of the trapped particle. There is also a provision for video imaging by a high speed CCD camera.

We have calibrated our system following standard procedures (Addas et al 2004). We have determined the calibration factor to be $1.906 \times 10^{-8} \mathrm{~m} / \mathrm{V}$ and trap stiffness to be $3.32 \mathrm{pN} / \mu \mathrm{m}$ at a laser power of $8 \mathrm{~mW}$ at the sample stage.

\section{Bacterial activity}

In order to characterize the antimicrobial activity of silver nanoparticles, we monitor the changes in the power spectral density (PSD) of a trapped bead in the bacterial suspension under investigation. The deviation in the PSD is taken into account. We have defined a term called activity factor (AF) which is a ratio of PSD of the bead in presence of active bacterial cells to that of the bead in the presence of inactive bacterial cells at the same cell count (Yogesha et al 2011). This is a parameter which has been defined for the first time to quantify collective bacterial activity. AF is inversely related to the antimicrobial action of any foreign particles that are added to the bacterial suspension. All measurements have been carried out at room temperature ( $295 \mathrm{~K})$.

\section{Results and discussion}

We have recorded position information data of a thermally fluctuating trapped bead at a scan rate $20 \mathrm{kHz}$. For each set of data, PSD is calculated (Schurr et al 1997; Addas et al 2004).

Figure 3 shows PSD values of the trapped particles suspended in (a) Pseudomonas aeroginosa, (b) E. coli and (c) Bacillus subtilis active suspensions in the presence of nanoparticles as a function of frequency. The data shown in this plot for all the samples are a normalized average of 25 data sets collected at the same temperature and under the same environmental conditions. As seen in figure 3, there is a change in PSD values, due to reduction in the bacterial activity with time. PSD values of control samples (without nanoparticles) do not show any such change on similar time scales.

Enhancement of PSD of a trapped bead immersed in a bacterial suspension when compared with that for a bead in water is larger. This is due to the hydrodynamic disturbances caused by the surrounding bacterial activity (Wu et al 2006) which consists of tumbling and swimming modes of action. On addition of an antimicrobial agent such as Ag nanoparticles, as in our work, the bacterial activity reduces with time 

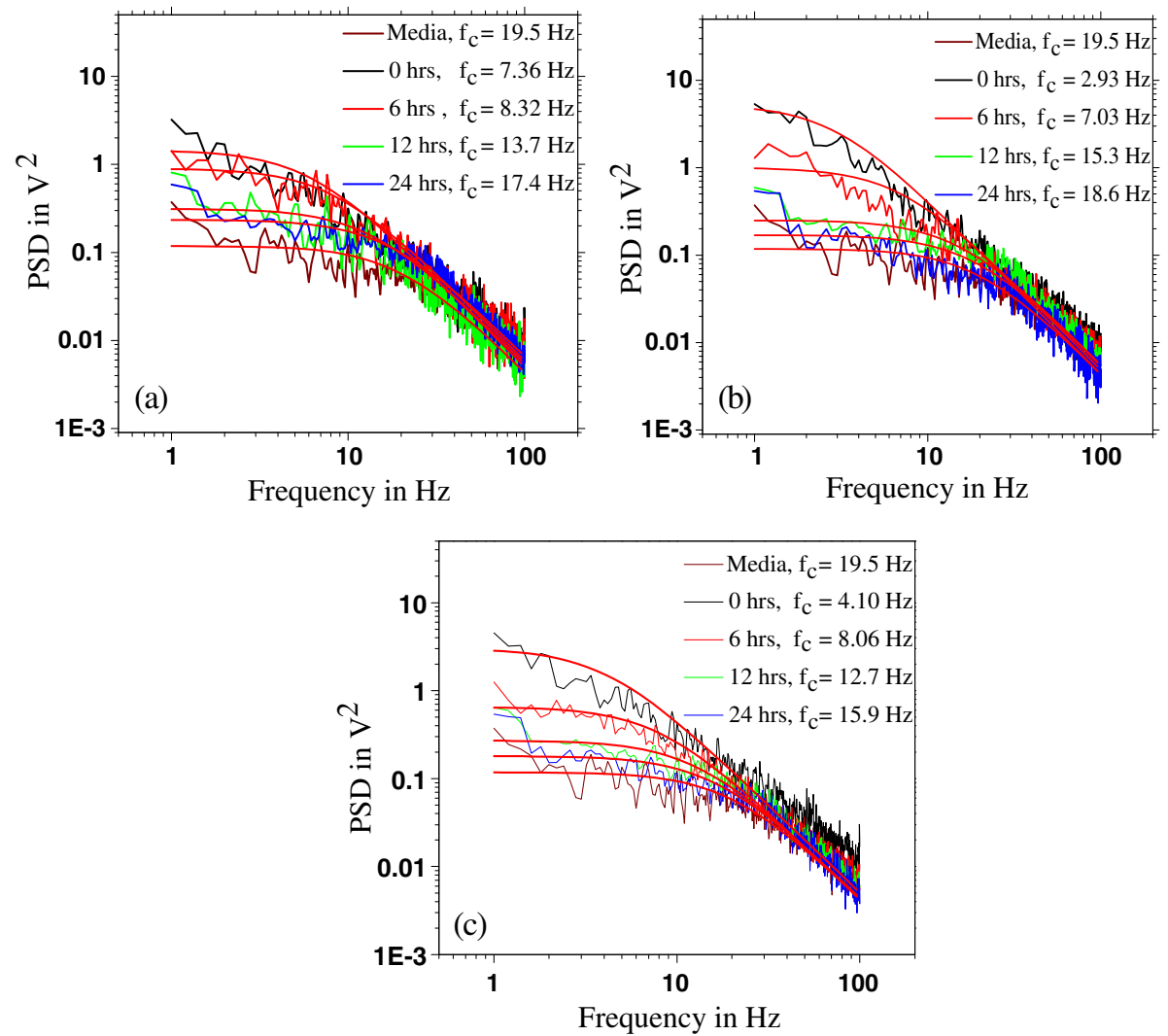

Figure 3. PSD data for (a) Pseudomonas aeroginosa, (b) E. coli and (c) Bacillus subtilis bacterial suspensions in presence of nanoparticles. Red lines are fits to data.
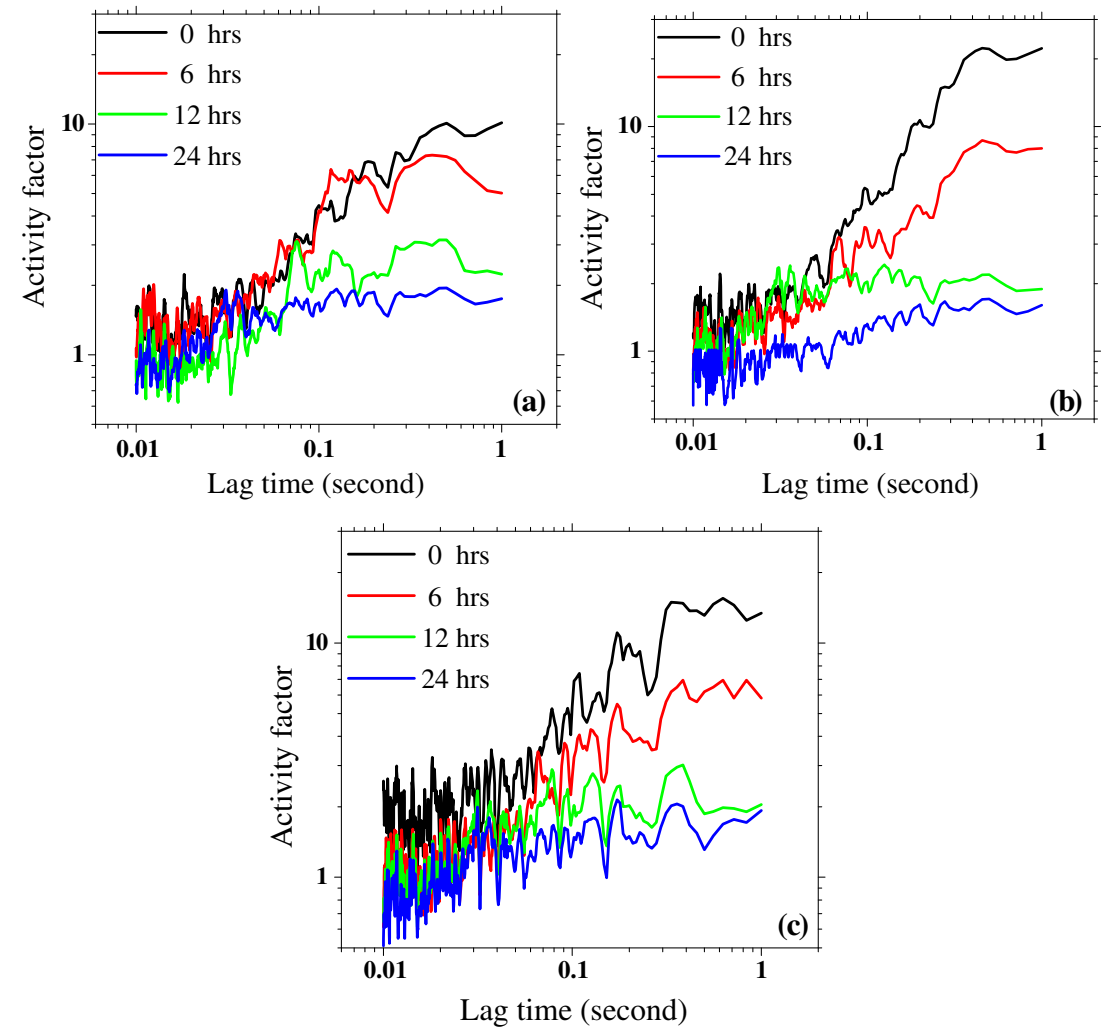

Figure 4. Activity factors of (a) Pseudomonas aeroginosa, (b) E. coli and (c) B. subtilis bacterial suspensions in presence of nanoparticles for different elapsed times. 
resulting in reduced hydrodynamic disturbance to the trapped bead, causing a fall in PSD values.

The corner frequency values of the PSD - frequency plots in figure 3 are obtained by fitting the PSD curve to an expression (Yogesha et al 2011)

$$
S_{\mathrm{x}}(f)=\frac{\left(\sqrt{2 \gamma k_{\mathrm{B}} T}+F_{\mathrm{B}}(f)\right)^{2}}{2 \pi^{2} \gamma^{2}\left(f_{\mathrm{c}}^{2}+f^{2}\right)},
$$

where $\gamma=6 \pi \eta a$ is the Stoke's drag coefficient, $\eta$ the viscosity of the medium, $a$ the radius of the bead, $k_{\mathrm{B}}$ the Boltzmann constant, $T$ the absolute temperature, $f_{\mathrm{c}}$ the corner frequency which is a measure of the trap stiffness, $f$ the frequency of fluctuations and $F_{\mathrm{B}}(f)$ the average force arising from the bacterial activity. It may be noted that this fit in addition, enables an estimation of the average bacterial force. It can be seen that $f_{\mathrm{c}}$ increases with time of contact due to the antibacterial action of the nanoparticles. It may be noted that the data has been fitted with stronger emphasis on the mid frequency range $(\sim 3 \mathrm{~Hz}$ to $50 \mathrm{~Hz})$. This has been done keeping in mind the high background noise at very low frequencies and also the fact that the corner frequency varies in the mid frequency range.

Figure 4 shows the activity factor as a function of lag time. This is measured for different times of contact of the antimicrobial agent with the bacterial population. The long lag time (low frequency) regime is most sensitive to the presence of bacteria whereas the small lag time (high frequency) regime is more representative of the thermal fluctuations in the medium. It is clear from the figure that the activity of bacteria reduces with increasing contact time. All our measurements are carried out with silver nanoparticle concentration of $0.66 \mu \mathrm{g} / \mathrm{ml}$.

We should add that in this method, we do not have a way of distinguishing between the effect of a reduced active population from a reduced average activity per bacterium. Nonetheless, our method enables a comparison between various antimicrobial agents on the same bacterial strain as well as a comparison between the antimicrobial action of the same agent on various bacterial strains.

\section{Conclusions}

Antimicrobial action of silver nanoparticles on bacterial strains of $P$. aeroginosa, E. coli and B. subtilis has been characterized using an optical tweezer based technique. This work illustrates the use of an optical tweezer based technique to monitor reduction in bacterial activity on exposure to an antimicrobial agent. In principle, thereby, an optical tweezer can be used as an effective tool for characterizing real time changes in bacterial activity under the action of different stimuli. However, the current study does not examine the signatures of various kinds of bacterial locomotion. Relating the activity factor to specific kinds of locomotion is an objective of future studies.

\section{Acknowledgement}

The authors wish to thank the DST, Govt. of India for a research grant (under Nano Mission).

\section{References}

Addas K M, Schmidt C F and Tang J X 2004 Phys. Rev. E70 021503

Alvarez M, Khoury J T, Schaaff G, Shafigullin M N, Vezmar I and Whetten R L 1997 J. Phys. Chem. B101 3706

Feng Q L, Wu J, Chen G Q, Cui F Z, Kim T N, Kim J O 2000 J. Biomed. Mater. Res. 52662

Lehninger A L and Nelson D L 1993 Principles of Biochemistry (New York: Worth Publishers) 2nd ed.

Rabinal M K et al 2012 Synthesis and characterization of silver nanoparticles (under preparation)

Raffi M, Hussain F, Bhatti T M, Akhter J I, Hameed A and Hasan M M 2008 J. Mater. Sci. Technol. 24192

Ruparelia J P, Chatterjee A K, Duttagupta S P and Mukherji S 2008 Acta Biomater. 4707

Schurr B, Gittes F, Mackintosh F C and Schmidt C F 1997 Macromolecules $\mathbf{3 0} 7781$

Wu M, Roberts J W, Kim S, Koch D K and Delisa M P 2006 Appl. Env. Microbiol. 724987

Yogesha, Nagesh B V, Raghu A, Sarbari Bhattacharya, Mohana D C and Sharath Ananthamurthy 2011 Int. J. Nano Science 10181 